\title{
A conceptual framework for understanding behavioral responses to HIREC
}

Andrew Sih, Pete C. Trimmer and Sean M. Ehlman

Department of Environmental Science and Policy, University of California, One Shields Avenue, Davis, CA 95616, USA

\begin{abstract}
Although animals vary substantially in their behavioral responses to human-induced rapid environmental change (HIREC), we are only beginning to develop theory to explain this variation. Signal detection theory predicts variation in responses to novel dangerous organisms (exotic predators or toxic prey) or exotic organisms that are safe but might appear dangerous (e.g., ecotourists). Models of dispersal and habitat use explain variation in ability to cope with habitat change (loss, fragmentation). Many models assume that organisms use one main cue axis to evaluate options. New models are needed to account for the use of multiple cues. A general framework that treats genes as cues that set a 'prior' that can be updated by experiences predicts genetic versus plastic responses to HIREC.
\end{abstract}

(C) 2016. This manuscript version is made available under the Elsevier user license http://www.elsevier.com/open-access/userlicense/1.0/ 
Organisms are experiencing novel environments associated with human-induced rapid environmental change (HIREC): habitat change [1,2], pollution $[3,4]$, exotic species $[5,6]$ human harvesting [7] and climate change [8,9]. While environmental variation per se is not new, human-induced changes are often on a larger spatial scale, and/or at a faster rate than in the past. For many animals, a critical element of their success (or lack of success) in coping with HIREC $[10,11]^{* *}$ is their initial behavioral response. Thus understanding behavior in novel, human-altered circumstances is an exciting and important challenge. Although the field has a rapidly growing number of case studies documenting the variation in behavioral responses to HIREC [11-14], it currently lacks a conceptual/theoretical framework [12*]. By and large, empirical studies are not testing predictions based on explicit theory, and results of these studies are not guiding the development of new theory to explain variation in observed responses. What the field needs is a conceptual/theoretical framework to: 1) organize hypotheses; 2) generate new questions and predictions; 3) guide empiricists on what to measure to better explain variation; and 4) ultimately, provide useful insights for management and conservation.

A key first step is to identify an approach that can potentially explain both why some animals exhibit immediate, adaptive responses to novel situations while other animals show responses that seem strikingly maladaptive. When an exotic predator invades, why do some naïve prey respond appropriately while others seem oblivious to the danger $[5,6]$ ? With global warming, why have some organisms exhibited a plastic shift to breed or migrate earlier in the spring, while others have not $[15,16]$ ? A common intuition is that organisms respond better to HIREC if the 'new', human-altered environment is not too different from what the organism saw in its evolutionary past [6]. Another general intuition is that flexible generalists that evolved to cope with variable environments should respond to HIREC better than specialists $[17,18]$. The ecological/evolutionary trap literature $[19,20]$ suggests that knowing 'cue-response systems' 
(how animals perceive, evaluate and respond to environmental cues) that underlie behavioral responses might be critical for understanding responses to both familiar and novel circumstances (also see [21,22]. With poor information, cue-response systems can lead animals to exhibit apparently maladaptive behaviors; the extent of this effect is likely to depend on the levels of environmental heterogeneity and autocorrelation [23]. Finally, if organisms survive initial encounters with novel situations, a common idea is that they should learn and eventually evolve to cope better with HIREC $[11,13]$.

To convert the above ideas into explicit, general models, we advocate using existing evolutionary ecology models to identify how past environmental conditions shaped current behavioral strategies and response systems that are examples of 'previously adaptive traits' (Fig 1). Although in the future, these previously adaptive traits can be altered (by learning and then evolution) to respond better to HIREC, a key question is how well the extant previously adaptive traits function when the environment first changes, before the animals have time to evolve. By contrasting new and past conditions, we can identify situations of mismatch where organisms are predicted to respond poorly. In brief, the approach: 1) uses existing behavioral/evolutionary ecology models to identify the previously adaptive traits ('decision variables') associated with each point in an environmental or cue-reliability parameter space, and 2) then extends those models to predict how animals with previously adaptive traits behave when they are moved to other regions of the parameter space; i.e., when they are exposed to novel environments associated with HIREC. Unlike traditional behavioral ecology theory that uses the optimality approach to explain adaptive behaviors, the new models aim to explain both adaptive and maladaptive behavioral responses to HIREC.

Earlier papers $[12,13]^{*}$ described how the approach outlined above can be applied to three major aspects of HIREC: 1) the use of signal detection theory to explain variation in responses of naïve prey to exotic predators; 2) adaptive plasticity theory to address variation in shifts in the seasonal timing of life histories (e.g., onset of breeding or migration) in response to 
climate change; and 3) adaptive learning models to predict variation in learning to adjust to HIREC. Here, we provide a condensed update on four additional, promising modeling frontiers for understanding variation in behavioral responses to HIREC.

Signal detection theory (SDT) can, in principle, generate predictions on behavioral responses to novel organisms (e.g., exotic predators or foods) as well as evolutionarily novel, humanassociated situations (e.g., urban habitat, automobiles, fishing gear). More generally, SDT analyzes responses when the state of the world (and thus the best response) is not perfectly known and information is received from frequency distributions of cue strengths $[24,25]$. Good vs bad foods or safe vs dangerous animals are easy to discriminate if their cue distributions are very different (e.g., dangerous animals are usually larger than safe ones), but difficult to discriminate if their cue distributions overlap heavily. In either case, the hypothesis is that an animal's decision when faced with a novel organism (e.g., potential food or predator) depends on its previously adaptive response threshold. For avoidance of danger, prey with a low response threshold will avoid most predators but also some situations that are not dangerous, whereas prey with a high response threshold will rarely err by avoiding non-dangerous situations but might ignore some dangerous predators. Sih [12] discussed how SDT models predict responses and fitness costs that accrue when prey encounter novel predators. An obvious prediction is that prey should tend to ignore novel predators (and thus suffer high mortality) if they are very different from familiar predators. SDT models show that errors should also depend on the reliability of previous options (animals 'trust' cues from novel options more if familiar cues were highly reliable), on the relative frequency of familiar good vs bad options, and on the previous costs and benefits of being bold versus timid (e.g. on how dangerous it was to not hide from past predators).

New work, adding state-dependence, suggests that novel organisms that are safe, but appear dangerous (e.g., ecotourists) can also substantially reduce 'prey' fitness; e.g., by inducing unnecessary hiding, ecotourists can reduce 'prey' food intake and ultimately, 
reproductive success. Intriguingly, models (and some data) suggest that even if ecotourists never directly kill prey, they can significantly increase prey mortality because hungry prey must take greater risks to feed and are thus more often killed by actual predators [26]*. Next steps in applying SDT to understanding responses to novel organisms could include consumer adoption (versus avoidance) of novel, high quality resources (e.g., crops, [27] ) or novel toxic prey (e.g., cane toads, [28]).

Dispersal and habitat use. Numerous studies have documented animal movements, dispersal and habitat settlement both in relatively undisturbed conditions and following habitat change, loss and fragmentation [29]. Some species appear to have adjusted well to habitat change, while others have fallen into ecological traps $[19,20]$. As with other aspects of HIREC, the field is only beginning to develop explicit theory to predict the relative success of different species in adjusting to habitat change. In principle, for animals using a patchy environment, the simple answer to the recurring question of whether to leave is: stay if the fitness benefit is better than one would expect from leaving. The devil, however, is in the uncertain details. For many organisms, adaptive dispersal and habitat use has always involved considerable uncertainty about key factors that should affect movement decisions: about the availability of different habitats, their quality, and costs of movement. Animals might have a decent assessment of their current situation, but can only guess (based on past experience or evolutionary history) what the future might hold in either their current or possible future patches []. After habitat change, their assessments of expected fitness for either decision are likely to be even less reliable. As a result, after habitat change, organisms might often disperse (i.e., continue to search) when they should settle, or vice versa.

Recent models have begun to address which mismatches between past and present environments following habitat change should be particularly damaging versus not as problematic. Relevant models vary from simple, two-habitat models [30] to more detailed 
simulation models of movement on simple landscapes [31-33] to even more detail-rich analyses of the evolution of patch boundary crossing rules on simulated, fractal landscapes with tens of thousands of grid cells [34]*. Notably, models to date differ in their predictions on the relative importance of factors that affect costs of habitat loss (e.g., on the relative importance of increased movement costs while searching versus reduced availability of high quality habitat); further work is needed to help to explain these differences and clarify predictions for empiricists to test.

Multiple cues. Almost all models for signal detection, adaptive plasticity or adaptive learning focus on one cue axis at a time. Real organisms, however, have multiple cues to choose among, and are known to use combinations of cues to make decisions. Multiple cues, often involving multiple sensory modes (e.g., visual, olfactory and auditory systems) are used in mate choice [35,36], habitat or host selection [37], food choice [38], in eliciting antipredator responses $[39,40]$, and in regulating the timing of life history switches [41]. Key questions include: 1) when and why do organisms use one cue as opposed to two or more? 2) If they use one cue - which one and why? And 3) if they use two or more cues, how do they combine information from cues (e.g., additively, and if so, how much they weigh each in importance)? Some may use nonlinear combinations - e.g., photoperiod must be sufficiently long before temperature starts to matter when preparing to migrate [42]. These issues are important for understanding responses to HIREC. For example, to understand life history responses to global warming, we need to know how and why organisms use photoperiod, temperature or other cues for regulating phenology. Hundreds of studies have quantified patterns in various taxa on what cues they use [41-43] but little general theory exists to explain the pattern of cue use from an adaptive framework.

Models emphasize that using multiple cues can increase reliability, reduce the chance of coding errors, or provide multiple, non-redundant messages $[39,44]$. Even if the multiple cues 
are redundant, the use of those cues should depend on the choice scenario - on the absolute and relative reliability of the cues, on correlations between the cues, on costs of sampling and assessment, and on the relative frequency of good and bad options [45]. The benefit of using multiple cues might be particularly high if the environment fluctuates [46]. But if there is no cost to using additional cues then theory suggests that they should be used [47]. While it is intuitive that using an increasing number of cues in assessing the environment is probably costly or involves cognitive limitations, we have a long way to go in understanding how and when multiple cues are integrated both from empirical and theoretical perspectives. Thus, in the HIREC theory context, an exciting step involves adding a second cue axis to signal detection [44], adaptive plasticity, and learning models to explore general predictions on how well organisms that have evolved different ways of using two cues should cope with various aspects of HIREC.

A general information framework. Several recent models have explored an even more general, information-based framework for understanding how the past shapes responses to current conditions (reviewed in $[48,49]^{*}$ ). One set of models treats "genes as cues". In these models, information about past environments is coded in adaptive genetic systems (including adaptive dominance and epistasis, and adaptive mutation and recombination rates) that combine, via the developmental system, with information from non-genetic, environmental cues to produce organismal traits $[48,50]$. The non-genetic cues could include transgenerational, epigenetic effects (transgenerational plasticity), and/or earlier experiences in the organism's lifetime (developmental plasticity). A Bayesian updating framework couches the same basic idea in terms of having a 'prior' estimate of the world which is updated when the organism is exposed to cues to produce a 'posterior' assessment $[49,51]$. These models predict that the relative importance of genetics, and different timescales of plasticity (transgenerational, developmental versus responses to current cues) in governing behavior should depend on their relative reliabilities as predictors of current conditions. If, for example, in the past, predation risk 
was always high, and new cues about risk have low reliability, organisms should evolve a relatively fixed, timid personality (even if cues suggest risk that might now be low), whereas in the opposite conditions, organisms should be more flexible, using current cues to gauge risk and behavior [52]. Note that rather than simply follow the quantitative genetic tradition of measuring the effects of genes $(G)$, environments $(E)$, their interaction $(G \times E)$ and the $G \times E$ correlation on traits, these information-based approaches predict their relative effects on traits, including some details (as noted above) on predicted genetic systems and developmental trajectories. In the HIREC context, a key issue is the degree to which organisms 'trust' new information and thus adjust to changing environments, as opposed to relying on their prior, now inaccurate, assessments. A still open niche involves adapting these information-based models to produce general predictions on how past information reliabilities and selection pressures should shape responses to novel conditions associated with HIREC.

Future Directions. We believe that we are at a stage similar to where behavioral ecology was $40+$ years ago when the field first developed optimality models to understand adaptive behavior in conditions that matched the organisms' evolutionary histories (e.g., optimal foraging, ideal free distribution, kin selection, hawk-dove, and parental investment models). These early models made many simplifying assumptions, but were nonetheless highly influential and useful in exemplifying a way of thinking, and in generating qualitative predictions that continue to guide the field. Work in subsequent decades added important aspects of reality (e.g., more strategic options, more complex tradeoffs, state-dependence, incomplete information) that allowed empiricists to more closely match models to specific systems. Now, to understand both adaptive and maladaptive responses to HIREC, we (as a field) are developing the $1^{\text {st }}$ order simple models to move from a largely descriptive phase to explicit models that help to both explain observed patterns and generate new predictions to test. In turn, these tests will guide the next stage of models. Previous decades of thinking in behavioral/ evolutionary ecology 
have provided the tools and expertise for us to move quickly to add key aspects of reality to $2^{\text {nd }}$ order models. It is an exciting - and important - time to be studying behavioral ecology in the modern world of HIREC.

Acknowledgements. This work was supported by a National Science Foundation grant to A. Sih (IOS 1456724) and a NSF Graduate Research Fellowship for S. Ehlman.

1. Fahrig L: Non-optimal animal movement in human-altered landscapes. Functional Ecology 2007, 21:1003-1015.

2. Elliot NB, Cushman SA, Macdonald DW, Loveridge AJ: The devil is in the dispersers: predictions of landscape connectivity change with demography. Journal of Applied Ecology 2014, 51:1169-1178.

3. Brodin T, Fick J, Jonsson M, Klaminder J: Dilute Concentrations of a Psychiatric Drug Alter Behavior of Fish from Natural Populations. Science 2013, 339:814-815.

4. Orci KM, Petroczki K, Barta Z: Instantaneous song modification in response to fluctuating traffic noise in the tree cricket Oecanthus pellucens. Animal Behaviour 2016, 112:187-194.

5. Carthey AJR, Banks PB: Naivete in novel ecological interactions: lessons from theory and experimental evidence. Biological Reviews 2014, 89:932-949.

6. Sih A, Bolnick DI, Luttbeg B, Orrock JL, Peacor SD, Pintor LM, Preisser E, Rehage JS, Vonesh JR: Predator-prey naivete, antipredator behavior, and the ecology of predator invasions. Oikos 2010, 119:610-621.

7. Heino M, Diaz Pauli B, Dieckmann U: Fisheries-induced evolution. Annual Review of Ecology, Evolution, and Systematics 2015, 46:461-480.

8. Dell Al, Pawar S, Savage VM: Temperature dependence of trophic interactions are driven by asymmetry of species responses and foraging strategy. Journal of Animal Ecology 2014, 83:7084. 
9. Sunday JM, Pecl GT, Frusher S, Hobday AJ, Hill N, Holbrook NJ, Edgar GJ, Stuart-Smith R, Barrett N, Wernberg $T$, et al.: Species traits and climate velocity explain geographic range shifts in an ocean-warming hotspot. Ecology Letters 2015, 18:944-953.

10. Hendry AP, Farrugia TJ, Kinnison MT: Human influences on rates of phenotypic change in wild animal populations. Molecular Ecology 2008, 17:20-29.

**11. Wong BBM, Candolin U: Behavioral responses to changing environments. Behavioral Ecology 2015, 26:665-673.

This paper (along with set of brief comments) is the key review to read to get up to speed on the topic of behavioral responses to HIREC. It identifies behavior (and, more specifically, behavioral plasticity) as playing a key role in the impact of HIREC on species' success. The authors consider various longer-term consequences in terms of population effects, inter-species interactions and ecological consequences and conclude that the field urgently needs a better grasp of the feedback loops at various levels of interaction.

*12. Sih A: Understanding variation in behavioural responses to human-induced rapid environmental change: a conceptual overview. Animal Behaviour 2013, 85:1077-1088.

This paper describes a conceptual framework for explaining variation in behavioral responses to HIREC. The authors illustrate the general framework using several modeling approaches (signal detection theory, adaptive plasticity theory, adaptive learning) that yield insights and predictions for broad classes of situations including exposure to novel predators or resources, and shifts in life history timing with climate change.

13. Sih A, Ferrari MCO, Harris DJ: Evolution and behavioural responses to human-induced rapid environmental change. Evolutionary Applications 2011, 4:367-387.

14. Tuomainen $U$, Candolin $U$ : Behavioural responses to human-induced environmental change. Biological Reviews 2011, 86:640-657.

15. Charmentier A, Gienapp P: Climate change and timing of avian breeding and migration: evolutionary versus plastic changes. Evolutionary Applications 2014, 7:1-14. 
16. Vatka E, Orell M, Rytkonen S: The relevance of food peak architecture in trophic interactions. Global Change Biology 2016, 22:1585-1594.

17. Blois JL, Zarnetske PL, Fitzpatrick MC, Finnegan S: Climate Change and the Past, Present, and Future of Biotic Interactions. Science 2013, 341:499-504.

18. Sol D, Sayol F, Ducatez S, Lefebvre L: The life-history basis of behavioural innovations. Philosophical Transactions of the Royal Society B-Biological Sciences 2016, 371:8.

19. Hale R, Coleman R, Pettigrove V, Swearer SE: REVIEW: Identifying, preventing and mitigating ecological traps to improve the management of urban aquatic ecosystems. Journal of Applied Ecology 2015, 52:928-939.

20. Robertson BA, Rehage JS, Sih A: Ecological novelty and the emergence of evolutionary traps. Trends in Ecology \& Evolution 2013, 28:552-560.

21. Greggor AL, Clayton NS, Phalan B, Thornton A: Comparative cognition for conservationists. Trends in Ecology \& Evolution 2014, 29:489-495.

22. Owen MA, Swaisgood RR, Blumstein DT: Contextual influences on animal decision-making: significance for behavior-based wildlife conservation and management. Integrative Zoology 2016, 11:in press.

23. Fawcett TW, Fallenstein B, Higginson AD, Houston AI, Mallpress DEW, Trimmer PC, McNamara JM, Modelling Anim Decisions G: The evolution of decision rules in complex environments. Trends in Cognitive Sciences 2014, 18:153-161.

24. Macmillan NA, Creelman CD: Detection Theory: A User's Guide edn 2nd. Mahwah, N.J.: Lawrence Erlbaum Associates; 2005.

25. Abbott KR, Sherratt TN: Optimal sampling and signal detection: unifying models of attention and speed-accuracy trade-offs. Behavioral Ecology 2013, 24:605-616.

*26. Geffroy B, Samia DSM, Bessa E, Blumstein DT: How Nature-Based Tourism Might Increase Prey Vulnerabiliy to Predators. Trends in Ecology \& Evolution 2015, 30:755-765.

This paper along with a set of comments discusses how apparently harmless ecotourists can have important negative or positive indirect impacts on organisms through effects on their behavior. 
Subsequent models attempt to identify conditions, including past selection pressures, that can explain when ecotourism might have particularly strong negative indirect effects.

27. Pearse IS, Harris DJ, Karban R, Sih A: Predicting novel herbivore-plant interactions. Oikos 2013, 122:1554-1564.

28. Doody JS, Soanes R, Castellano CM, Rhind D, Green B, McHenry CR, Clulow S: Invasive toads shift predator-prey densities in animal communities by removing top predators. Ecology 2015, 96:2544-2554.

29. Chaine AS, Clobert J: Dispersal. In Behavioural responses to a changing world. Mechanisms and consequences. Edited by Candolin U, Wong BBM: Oxford University Press; 2012:63-79.

30. Stamps JA, Krishnan VV, Reid ML: Search costs and habitat selection by dispersers. Ecology 2005, 86:510-518.

31. Enfjall K, Leimar O: The evolution of dispersal - the importance of information about population density and habitat characteristics. Oikos 2009, 118:291-299.

32. Bocedi G, Travis JMJ: Models of Dispersal Evolution Highlight Several Important Issues in Evolutionary and Ecological Modeling. American Naturalist 2016, 187:143-150.

33. Travis JMJ, Mustin K, Barton KA, Benton TG, Clobert J, Delgado MM, Dytham C, Hovestadt T, Palmer SCF, Van Dyck $\mathrm{H}$, et al.: Modelling dispersal: an eco-evolutionary framework incorporating emigration, movement, settlement behaviour and the multiple costs involved. Methods in Ecology and Evolution 2012, 3:628-641.

*34. Martin AE, Fahrig L: Matrix quality and disturbance frequency drive evolution of species behavior at habitat boundaries. Ecology and Evolution 2015, 5:S792-S800.

This paper describes a particularly detail-rich, individual-based model of animal movements evolved to be adaptive in a range of natural landscapes that differ in patch size and quality and matrix quality. The authors then investigate how well these evolved strategies cope with habitat loss and fragmentation. The analysis illustrates how models can provide insights on situations that are particularly vulnerable to HIREC. 
35. Taff CC, Steinberger D, Clark C, Belinsky K, Sacks H, Freeman-Gallant CR, Dunn PO, Whittingham LA: Multimodal sexual selection in a warbler: plumage and song are related to different fitness components. Animal Behaviour 2012, 84.

36. Girard MB, Elias DO, Kasumovic MM: Female preference for multi-modal courtship: multiple signals are important for male mating success in peacock spiders. Proceedings of the Royal Society B-Biological Sciences 2015, 282:10.

37. Proffit M, Khallaf MA, Carrasco D, Larsson MC, Anderson P: 'Do you remember the first time?' Host plant preference in a moth is modulated by experiences during larval feeding and adult mating. Ecology Letters 2015, 18:365-374.

38. Rubi TL, Stephens DW: Should receivers follow multiple signal components? An economic perspective. Behavioral Ecology 2016, 27:36-44.

39. Munoz NE, Blumstein DT: Multisensory perception in uncertain environments. Behavioral Ecology 2012, 23:457-462.

40. Mella VSA, Banks PB, McArthur C: Negotiating multiple cues of predation risk in a landscape of fear: what scares free-ranging brushtail possums? Journal of Zoology 2014, 294:22-30.

41. Baskin CC, Baskin JM: Seeds: Ecology, Biogeography, and Evolution of Dormancy and Germination: Academic Press; 2014.

42. Dawson A: Control of the annual cycle in birds: endocrine constraints and plasticity in response to ecological variability. Philosophical Transactions of the Royal Society B-Biological Sciences 2008, 363:1621-1633.

43. Buchanan $\mathrm{KL}$, Partecke $\mathrm{J}$ : The endocrine system: can homeostasis by maintained in a changing world? In Behavioural responses to a changing world. Edited by Candolin U, Wong BBM: Oxford University Press; 2012:32-45.

44. Munoz NE: Multimodal integration in uncertain environments: a quantitative model and field experiments with Yellow-bellied marmots (Marmota flaviventris). PhD dissertation, University of California at Los Angeles; 2015.

45. Fawcett TW, Johnstone RA: Optimal assessment of multiple cues. Proceedings of the Royal Society B-Biological Sciences 2003, 270:1637-1643. 
46. Bro-Jorgensen J: Dynamics of multiple signaling systems: animal communication in a world in flux. Trends in Ecology Evolution 2009, 25:292-300.

47. McNamara JM, Dall SRX: Information is a fitness enhancing resource. Oikos 2010, 119:231-236.

*48. Dall SRX, McNamara JM, Leimar O: Genes as cues: phenotypic integration of genetic and epigenetic information from a Darwinian perspective. Trends in Ecology \& Evolution 2015, 30:327333.

The authors review recent models that develop the idea that genetics, epigenetics and plasticity can be integrated via the developmental system to provide an adaptive explaination for their roles in shaping phenotypes through their relative value as sources of information about current or future environments. An exciting next step involves adapting this general approach to explain how these factors shape responses to HIREC.

49. Stamps JA, Frankenhuis WE: Bayesian Models of Development. Trends in Ecology \& Evolution 2016, 31:260-268.

50. Leimar O, McNamara JM: The Evolution of Transgenerational Integration of Information in Heterogeneous Environments. American Naturalist 2015, 185:E55-E69.

51. Stamps JA, Krishnan VV: Combining Information from Ancestors and Personal Experiences to Predict Individual Differences in Developmental Trajectories. American Naturalist 2014, 184:647657.

52. Sih A: Prey uncertainty and the balancing of antipredator and feeding needs. American Naturalist 1992, 139:1052-1069.

53. McNamara JM, Houston Al. Integrating function and mechanism. Trends in Ecology \& Evolution 2009, 24: 670-675. 


\section{Figure Legend.}

Figure 1. The HIREC modeling pipeline provides a general approach for modelling HIREC effects over time by examining discrete stages of change. In the top row, models analyze optimal behaviors (decision variables) under uncertainty and outcomes for 'pre-HIREC' conditions. Environmental changes can be modeled simply by changing parameters. This approach allows the examination of post-HIREC behavioral and fitness outcomes before and after learning has occurred (lines 2 and 3). The final line assumes that the system re-optimizes through evolution to deal with the new conditions appropriately, perhaps even requiring a change of modelling approach (which is likely to be difficult and require meta-modelling; cf. Level 3 questions in [50]). By comparing behavior and fitness outcomes before and after HIREC, we can quantify fitness costs (or benefits) of HIREC initially, then after animals have learned about the novel environment, and finally, after subsequent evolution. 